\title{
Optimization of Bistability in Nonlinear Chalcogenide Fiber Bragg Grating for All Optical Switch and Memory Applications
}

\author{
Elham Yousefi ${ }^{\mathrm{a}}$, Mohsen Hatami ${ }^{\mathrm{a}, \mathrm{b}}$, and Sajjad Dehghani ${ }^{\mathrm{c}, *}$

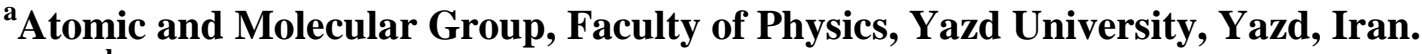 \\ ${ }^{b}$ Physics Department, Shiraz University of Technology, Shiraz, Iran. \\ ${ }^{c}$ Faculty of Advanced Technologies, Shiraz University, Shiraz, Iran. \\ *Corresponding Author's Email: sdehghani@shirazu.ac.ir
}

Received: Jun. 20, 2016, Revised: Jul. 27, 2016, Accepted: Aug. 20, 2016, Available Online: Jan. 28 , 2017

DOI: 10.18869 /acadpub.ijop.11.1.49

\begin{abstract}
We solve the coupled mode equations governing the chalcogenide nonlinear fiber Bragg gratings (FBGs) numerically, and obtain the bistability characteristics. The characteristics of the chalcogenide nonlinear FBGs such as: switching threshold intensity, bistability interval and on-off switching ratio are studied. The effects of FBG length and its third order nonlinear refractive index on FBG characteristics are investigated. We obtain an interesting result that independent of the third order nonlinear refractive index; there exists an optimum FBG length of about $6 \mathrm{~mm}$ at which the on-off switching ratio becomes maximized. It is also found that by increasing the nonlinearity, the maximum value of on-off switching ratio decreases. The results of this paper can be mainly used for designing alloptical switches and memories.
\end{abstract}

KEYWORDS: Nonlinear fiber Bragg grating (FBG), optical bistability, bistability characteristics, all optical memory, all optical switch.

\section{I.INTRODUCTION}

Because of growing fiber Bragg grating (FBG) applications in optical communication systems [1-3] and optical fiber sensors [4], they will play a crucial role in future all-optical communication [5]. A considerable advantage of nonlinear FBGs is that they can be designed as all optical switches [6-8] and optical bistable devices [9-11]. The study of optical switching and optical bistability has been started since 1979 [12]. Using common silica FBGs, these phenomena require high peak intensity in the order of $\mathrm{GW} / \mathrm{cm}^{2}$ [3]. Until recently, when using chalcogenide FBGs, the required peak intensity for optical bistability has been decreased 1000 times, because of their higher third order nonlinear coefficient compared to the silica one $[13,14]$. Therefore, shorter length is needed to create high quality bistability and switching phenomena when chalcogenide FBG is used.

In the present paper, the optical bistability in chalcogenide fiber Bragg gratings with third order nonlinearity is studied numerically. The effects of fiber length and third order nonlinear refractive index on switching threshold intensity, bistability interval and ratio of on-off switching will be studied in section 3. Finally, optimization of switching and bistability with regards to input intensity, FBG length and nonlinear coefficient of chalcogenide materials is carried out. The obtained results are helpful for future design of all-optical memories and switches.

\section{THEORY AND NUMERICAL Calculations}

Consider a lossless uniform FBG with length of $L$ which is shown in Fig. 1. 
The refractive index of a uniform fiber Bragg grating as a function of position, $z$ and electric field in the FBG, $E$ can be written as [15]:

$n(z)=n_{0}+n_{1} \cos \left(\frac{2 \pi}{\Lambda} z\right)+n_{2}|E|^{2}$,

where $\Lambda$ is the FBG period, $n_{0}, n_{1}$ and $n_{2}$ are the linear refractive index, refractive index modulation amplitude, and third order nonlinear refractive index coefficient, respectively.

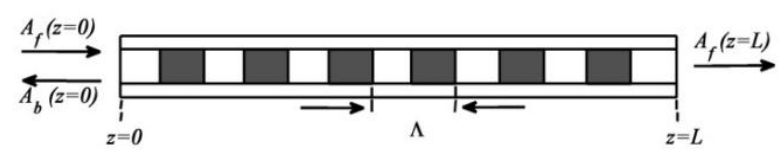

Fig. 1. The schematic diagram of the FBG with length of $\mathrm{L}$. The forward and backward waves are shown.

By combining forward and backward waves, the electric field in the FBG can be described as [15]:

$$
\begin{aligned}
E(\mathbf{r}, t)= & \frac{1}{2} F(x, y)\left[A_{f}(z, t) e^{i \beta_{B} z}\right. \\
& \left.+A_{b}(z, t) e^{-i \beta_{B} z}\right] e^{-i \omega_{0} t}+c . c .,
\end{aligned}
$$

where $A_{f}$ and $A_{b}$ are the slowly varying amplitude of forward and backward waves respectively and $\omega_{0}$ is the carrier frequency. $\mathrm{F}(\mathrm{x}, \mathrm{y})$ is the transverse variations of the two counter-propagating waves.

In this paper, we take continuous wave $(\mathrm{CW})$ limit by neglecting the time derivatives as it is discussed in [16]. By substitution of Eqs. (1) and (2) into the Maxwell's wave equations and considering slowly varying amplitude approximation, the nonlinear coupled mode equations for both forward and backward wave amplitudes can be obtained as follows [15, 16]:

$$
\begin{aligned}
\frac{\partial A_{f}}{\partial z}+\frac{\alpha}{2} A_{f}= & i \delta A_{f}+i \kappa A_{b} \\
& +i \gamma\left(\left|A_{f}\right|^{2}+2\left|A_{b}\right|^{2}\right) A_{f},
\end{aligned}
$$

$$
\begin{aligned}
-\frac{\partial A_{b}}{\partial z}+\frac{\alpha}{2} A_{b}= & i \delta A_{b}+i \kappa A_{f} \\
& +i \gamma\left(\left|A_{b}\right|^{2}+2\left|A_{f}\right|^{2}\right) A_{b},
\end{aligned}
$$

where $\alpha$ represents the FBG loss which is neglected here, $\delta, \kappa$ and $\gamma$ account for detuning from the Bragg wavelength, coupling coefficient, and nonlinear coefficient, respectively.

By applying $4^{\text {th }}$ order Rung-Kutta numerical method, we solve the aforementioned coupled mode nonlinear equations numerically.

As it is discussed in [2, 5, 17], we perform these calculations from the end of the FBG to its beginning using boundary conditions of $A_{f}(z=L)=A_{f L}, A_{b}(z=L)=0$, where $A_{f L}$ is an adjustable value for better simulation. The results are shown in Figures 2-10.

\section{RESULTS AND DISCUSSION}

As shown in Fig. 2 a typical optical bistability diagram contains three branches: low, medium, and high branch which are shown by $\mathrm{AB}, \mathrm{BE}$ and $\mathrm{ED}$ lines, respectively. The middle branch, i.e. BE, is unstable and reaching to it is almost impossible in practice. Bistability interval, on-off switching ratio, and switching threshold intensity are three important parameters of optical bistability, as presented in Fig. 2. The bistability interval is the interval of input intensities in which the bistability diagram has two stable outputs. The on-off switching ratio is the amount of change in the output intensity when switching on is occurring, and the switching threshold intensity is the minimum input intensity required for switching on i.e. for transition from low branch to the high branch in bistability curve. Typical values of parameters used in this paper are shown in Table 1. We study the effect of some of these parameters on bistability characteristics such as switching threshold intensity, bistability interval and ratio of on-off switching. 
Table 1. Typical data used in this work

$\begin{array}{lll}\lambda_{B}=1550 \mathrm{~nm} & n_{0}=2.54 & n_{2}=220 n_{2 \text { silica }} \\ \lambda=1550.015 \mathrm{~nm} & n_{1}=1.5 \times 10^{-4} & n_{2 \text { silica }}=0.273 \times 10^{-19} \mathrm{~m}^{2} / W_{[19]}\end{array}$

$\Lambda=3.1631 \times 10^{-7} \mathrm{~m}$

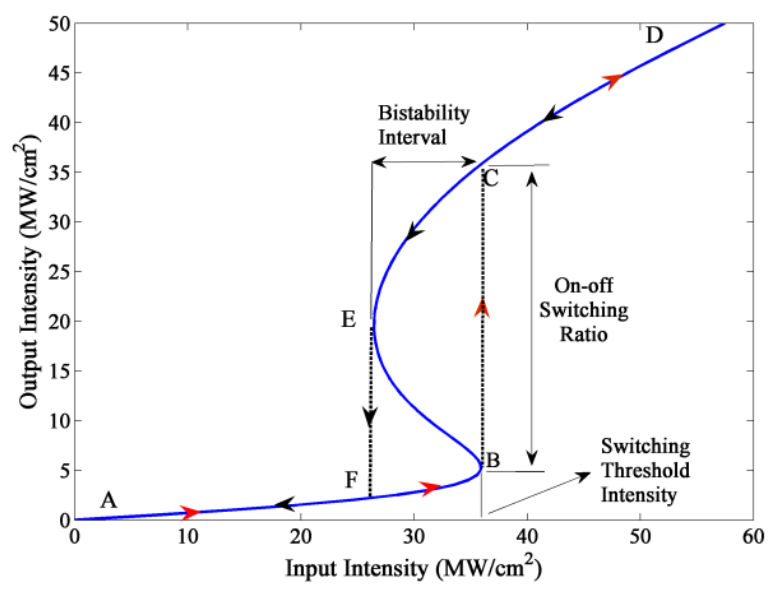

Fig. 2. Typical optical bistability diagram.

\section{A. Effect of fiber length on optical bistability}

Since FBG length is normally introduced by $L=N_{L} \Lambda$, where $N_{L}$ is the number of FBG periods, we follow the same notation and use $N_{L}$ instead of $L$. The effect of FBG period on bistability diagram was studied. The range of FBG period was $15000-23000$ corresponding to FBG lengths of $4.7-7.9 \mathrm{~mm}$.

The results are depicted in Fig. 3. The lowest value of the number of FBG periods (FBG's length) is chosen to be near to the onset of bistability. This figure shows that increasing the number of the FBG periods, improves the bistability and also there is a minimum length below which there is no bistability. There are two possible reasons for this behavior. First, by increasing the length, nonlinearity increases. Second, by increasing the length, the output intensity is amplified even greater than the input intensity of the FBG which intensifies the nonlinearity effect as can be seen in Fig.4. In this figure the intensity of transmitted light is presented as a function of position, for $n_{2}=220 n_{2 s i l i c a}$. The intensity of input wave is $1 \mathrm{MW} / \mathrm{cm}^{2}$. As the intensity of field increases in the fiber so does the nonlinear effect. Due to the nonlinear effect and reflecting from periodic structure of Bragg grating, the intensity of transmitted field increases in the fiber. It is an interesting phenomenon that the intensity of filed in the FBG can be greater than the input intensity. Due to these two main causes, the nonlinearity is increased, which leads to improvement of the bistability and as a result the switching threshold intensity is decreased. Fig. 3 shows the switching threshold intensity and the bistability interval with regard to the length of fiber. This figure indicates the improvement of bistability, i.e. decreasing the switching threshold by increasing the length.

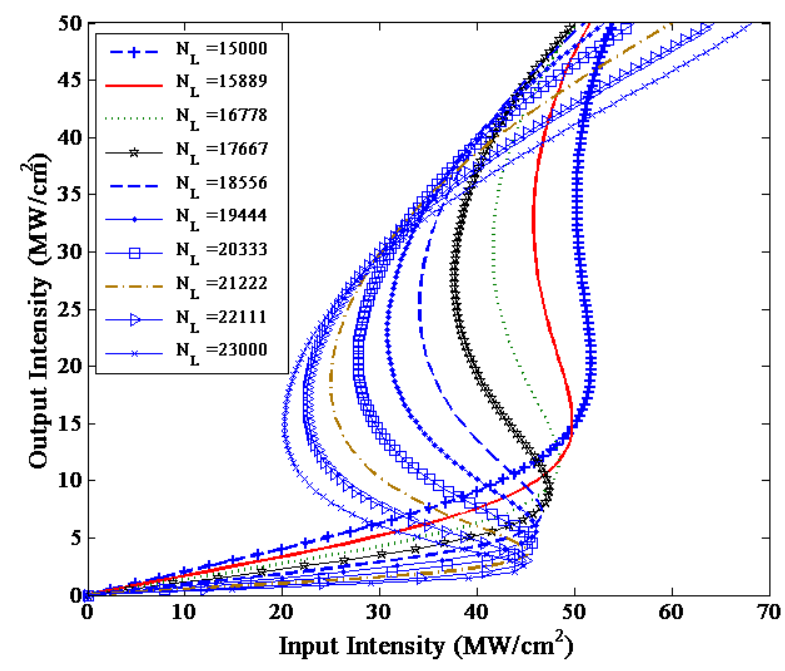

Fig. 3. The effect of number of FBG periods on optical bistability characteristics.

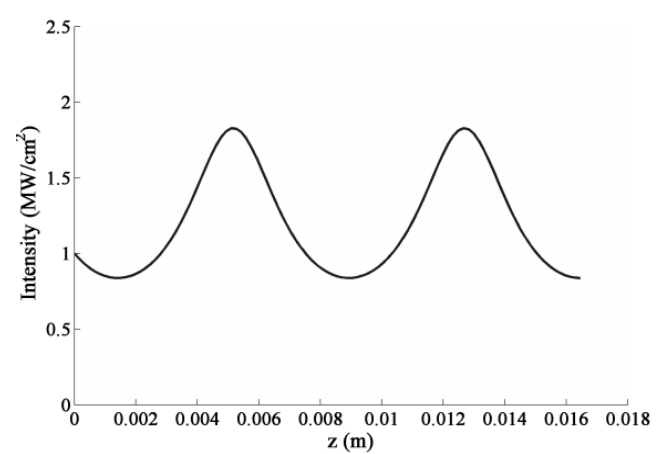

Fig. 4. The intensity of light transmitted in the FBG as a function of position.

The on-off switching ratio as a function of number of FBG periods is shown in Fig. 6. The result of this figure is considerable because it shows that the on-off switching ratio diagram has a maximum value at a specific number of FBG periods. In other 
words there is an optimum FBG length at which the switching reaches its maximum value and it is important for designing FBGs with better switching behavior. The maximum of on-off switching ratio is almost occurred at $N_{L}=18800$ corresponding to $L=5.9 \mathrm{~mm}$.

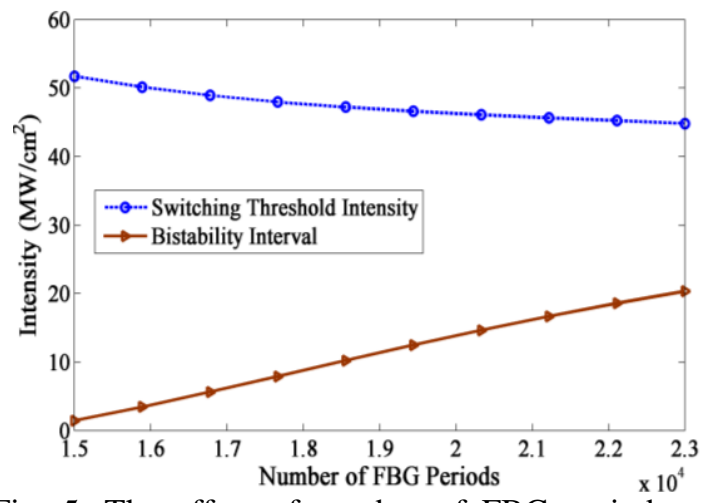

Fig. 5. The effect of number of FBG periods on bistability interval and switching threshold intensity.

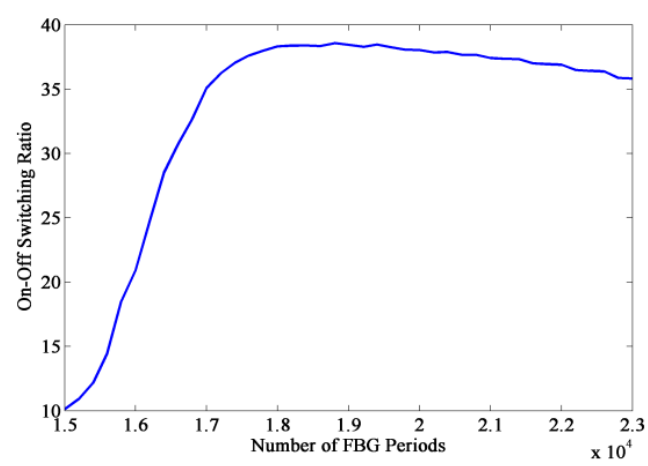

Fig. 6. The on-off switching ratio as a function of number of FBG periods.

\section{B. Effect of third order nonlinear refractive index on optical bistability}

Chalcogenide materials have a wide range of nonlinearity ranging from about 100 to 1000 times of the fused silica as indicated in Table 2. The bistability characteristics depend on third order nonlinear refractive index. Naturally by increasing the third order nonlinear refractive index the bistability improves and hence we studied its effect on bistability characteristic numerically. The results of our simulations are illustrated in Fig. 7. Each curve of this figure refers to a specific value of third order nonlinear refractive index which is selected from Table 2. The results evidently illustrate that increasing the nonlinearity improves the desired bistability parameters. The connection between the parameters and bistability are presented in Fig. 8. This figure indicates the reduction of switching threshold due to increment of the third order nonlinear refractive index, as expected and desired. In addition, it shows that the bistability interval and on-off switching ratio decrease when the third order nonlinear refractive index increases. Therefore depending on the FBG application i.e. optical switch or optical bistable device, suitable chalcogenide material can be selected.

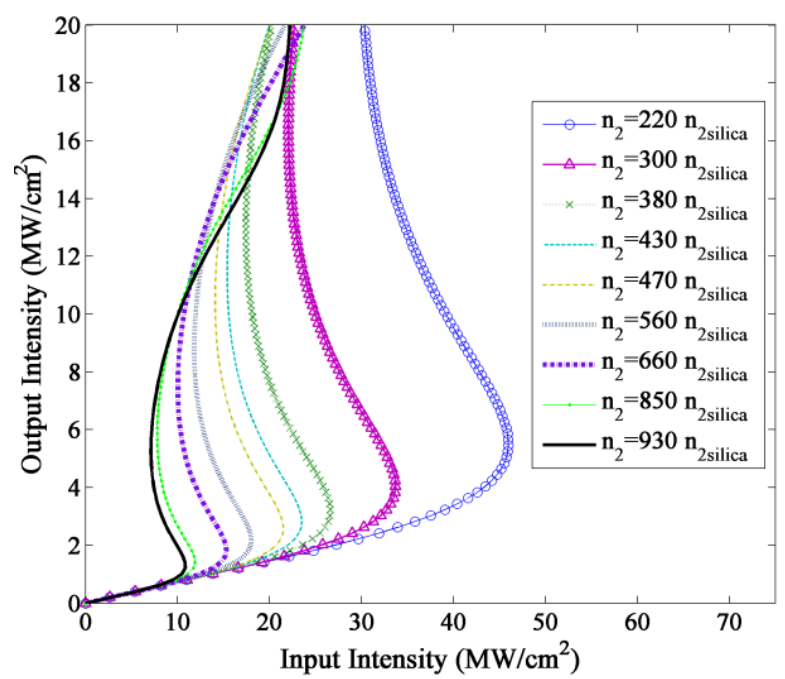

Fig. 7. Optical bistability curves for nine chalcogenide glasses of different third order nonlinear refractive indices.

Table 2. $n_{0}$ and $n_{2}$ parameters of chalcogenide glasses [18]

\begin{tabular}{|c|c|c|}
\hline Glass & $n_{0}$ at $1.55 \mu \mathrm{m}$ & $n_{2} / n_{2 \text { silica }}( \pm 15 \%)$ \\
\hline $\bar{A} \mathbf{s}_{40} \mathbf{S}_{60}$ & 2.45 & 220 \\
\hline $\mathbf{A s}_{40} \mathbf{S}_{40} \mathbf{S e}_{20}$ & 2.55 & 300 \\
\hline $\mathrm{As}_{40} \mathbf{S}_{50} \mathrm{Se}_{10}$ & 2.49 & 380 \\
\hline $\mathbf{A s}_{40} \mathbf{S}_{30} \mathbf{S e}_{30}$ & 2.62 & 430 \\
\hline $\mathrm{As}_{25} \mathrm{~S}_{55} \mathrm{Te}_{20}$ & 2.52 & 470 \\
\hline $\mathbf{A s}_{40} \mathbf{S}_{10} \mathbf{S e}_{50}$ & 2.76 & 560 \\
\hline$A s_{30} S_{e}$ & 2.81 & 660 \\
\hline $\mathrm{As}_{40} \mathrm{Se}_{55} \mathrm{Cu}_{5}$ & 2.93 & 850 \\
\hline $\mathrm{As}_{40} \mathrm{Se}_{60}$ & 2.81 & 930 \\
\hline
\end{tabular}

\section{Optimum fiber length for various third order nonlinear coefficients}

The on-off switching ratio versus the number of FBG periods for nine chalcogenide glasses of different third order nonlinear refractive indices is plotted in Fig. 9. It may be expected that by increasing the third order refractive index, we have a lower optimum length. However, the results show otherwise. We 
found that the optimum length for maximizing the on-off switching ratio is nearly independent of $\mathrm{n}_{2}$. This optimum FBG length is about $6 \mathrm{~mm}$ as shown in Fig. 10.

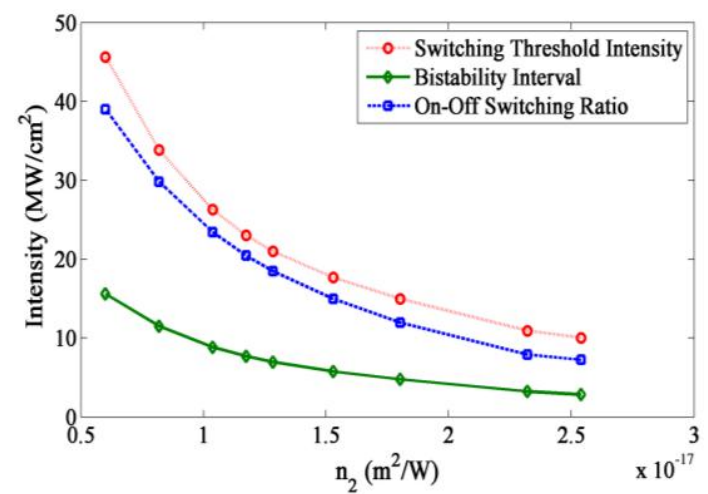

Fig. 8. The diagrams of switching threshold, bistability interval and on-off switching ratio versus third order nonlinear refractive index.

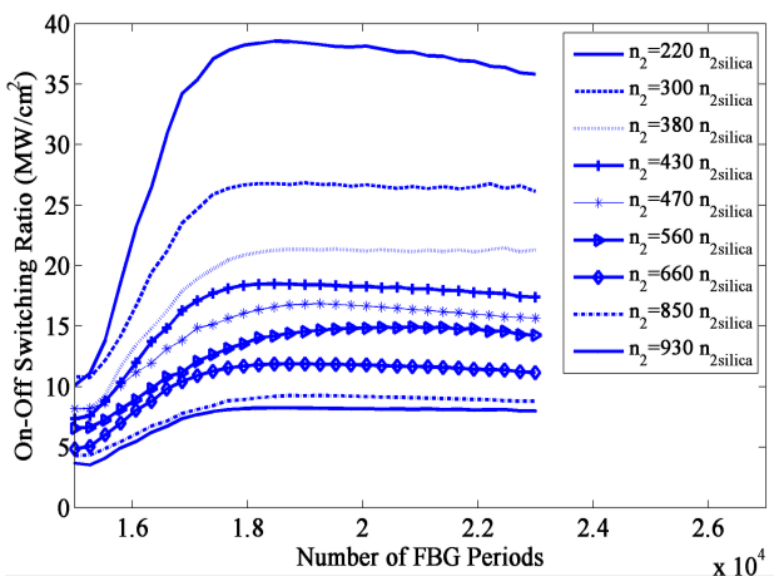

Fig. 9. The on-off switching ratio versus number of FBG periods for nine chalcogenide glassess of different third order refractive indicies .

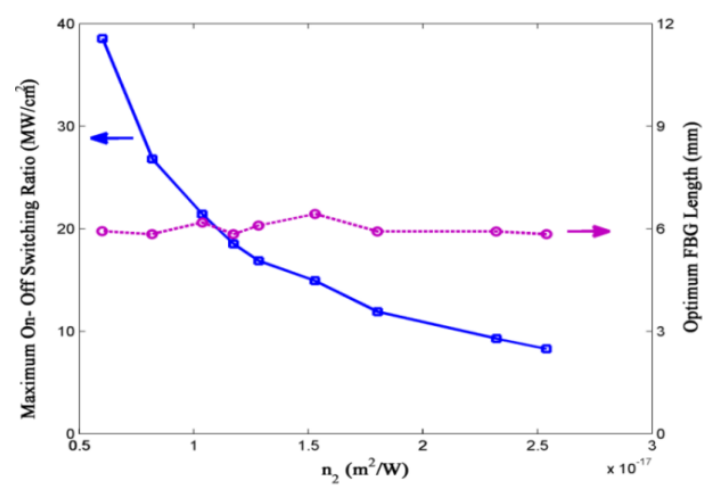

Fig. 10. The maximum on-off switching ratio and optimum FBG length versus third order refractive index of chalcogenide glassess.

Fig. 9 also indicates that increasing the third order refractive index makes the on-off switching ratio more stable and flatted above the optimum length. These results are useful and important for designing optical switches and memories.

As seen in Fig. 10, by increasing the third order nonlinear refractive index the maximum on-off switching ratio is decreased however the optimum FBG length is almost constant. In Table 3, the Suitable range of bistability parameters for optical switch and memory applications is qualitatively shown. As shown in this Table the high range of on-off switching ratio as well as low range of switching threshold, is suitable for both optical switch and memory devices. However the desired value of bistability interval depends on application. For switching application low range of bistability interval is required, while, the bistability interval of optical memories should be sufficiently high.

Tabel 3. Suitable range of bistability parameters for optical switch and memory applications.

\begin{tabular}{lcc}
\multicolumn{3}{c}{ memory applications. } \\
\hline $\begin{array}{l}\text { Bistability } \\
\text { characteristic }\end{array}$ & Low & High \\
\hline $\begin{array}{l}\text { On-off switching } \\
\text { ratio }\end{array}$ & - & $\begin{array}{c}\text { Optical switch and } \\
\text { optical memory }\end{array}$ \\
\hline $\begin{array}{l}\text { Switching } \\
\text { threshold }\end{array}$ & $\begin{array}{c}\text { Optical switch and } \\
\text { optical memory }\end{array}$ & --- \\
\hline $\begin{array}{l}\text { Bistability } \\
\text { interval }\end{array}$ & Optical switch & Optical memory \\
\hline
\end{tabular}

\section{IV.CONCLUSION}

By numerical calculations, we solved the nonlinear coupled mode equations which govern the pulse propagation through FBG and derived the bistability diagram. We investigated the effects of two important parameters: FBG length and third order nonlinear refractive index. Finally by comparing the bistability diagrams the following results are found: First, the switching threshold is decreased by increasing FBG length as well as third order refractive index. Second, the bistability interval can be increased by increasing the FBG length or by decreasing the third order refractive index. Third, the on-off switching ratio is decreased by increasing the third order nonlinear refractive index. Finally, independent of the third order refractive index, an optimum FBG length of about $6 \mathrm{~mm}$ at which the on-off switching ratio is maximized was found. These 
results are the base of planning and designing all-optical integrated devices such as flipflops.

\section{REFERENCES}

[1] B.A. Daniel and G.P. Agrawal, "Design of phase-switched two-input Kerr flip-flops," J. Opt. Soc. Am. B, Vol. 29, No. 9, pp. 22882296, 2012.

[2] E. Yousefi and M. Hatami, "All optical self signal processing using chalcogenide nonlinear fiber Bragg grating," Optik, Vol. 125, No. 22, pp. 6637-6640, 2014.

[3] H. Lee and G.P. Agrawal, "Nonlinear switching of optical pulses in fiber Bragg gratings," IEEE J. Quantum Electron. Vol. 39, No. 3, pp. 508-515, 2003.

[4] A. Othonos and K. Kalli, Fiber Bragg Gratings: Fundamentals and Applications in Telecommunications and Sensing, Artech House, 1999.

[5] E. Yousefi, M. Hatami, and A. Torabi Jahromi, "All-optical ternary signal processing using uniform nonlinear chalcogenide fiber Bragg gratings," J. Opt. Soc. Am. B, Vol. 32, No. 7, pp. 1471-1478, 2015.

[6] S. Radic, N. George and G.P. Agrawal, "Theory of low-threshold optical switching in nonlinear phase-shifted periodic structures," J. Opt. Soc. Am. B, Vol. 12, No. 4, pp. 671-680, 1995.

[7] N.G.R. Broderick, D.J. Richardson, and M. Ibsen, "Nonlinear switching in a 20-cm-long fiber Bragg grating," Opt. Lett. Vol. 25, No. 8, pp. 536-538, 2000.

[8] H. Zoweil and J.W.Y. Lit, "Bragg grating with periodic non-linearity as optical switch," Opt. Commun. Vol. 212, No. 1-3, pp. 57-64, 2002.

[9] N.G.R. Broderick, "Bistable switching and multiple gap-soliton formation in a fiber Bragg grating," Opt. Commun. Vol. 148, pp. 90-94, 1998.

[10] Y. Peng, K. Qui, B. Wu, and S. Ji, "Study on characteristics of optical bistable devices based on fiber Bragg grating," SPIE-OSAIEEE Asia Commun. and Photonics Conf and Exhibition, SPIE, Vol. 7630, 2009.

[11]Ch. X. Shi, "Optical bistability in reflective fiber gratings," IEEE J. Quantum Electron. Vol. 31, No. 11, pp. 2037-2043, 1995.
[12] H.G. Winful, J.H. Marburger, and E. Garmire, "Theory of Bistability in Nonlinear Distributed feedback structures," Appl. Phys. Lett. Vol. 35, No. 5, pp. 379-381, 1979.

[13] Y. Yosia and S. Ping, "Double Optical Bistability and its Application in Nonlinear Chalcogenide-Fiber Bragg Gratings," Phys. B, Vol. 394, No. 2, pp. 293-296, 2007.

[14] Y. Yosia and S. Ping, "Optical bistability in periodic media with third fifth- and seventhorder nonlinearities," J. Lightwave Technol. Vol. 25, No. 3, pp. 875-882, 2007.

[15] G.P. Agrawal, Applications of Nonlinear Fiber Optics, Academic Press, 2001.

[16] A.F. de Morais Neto, C.S. Sobrinho, A.F.G. Furtado Filho, J.W.M. Menezes, and A.S.B. Sombra, "Ultrashort pulse reflection through nonlinear fiber Bragg gratings," ITS Conf. IEEE, pp.739-742, 2006.

[17] J.F. Tian, "Investigation on the bistability of linearly chirped nonlinear Bragg gratings," Int. J. Light Electron. Opt. Vol. 122, No. 15, pp. 1381-1383, 2011.

[18] J.M. Harbold, F.O. Ilday and F.W. Wise, "Highly nonlinear As-S-Se glasses for alloptical switching," Opt. Lett. Vol. 27, No. 2, pp. 119-121, 2002.

[19]G.P. Agrawal, Nonlinear Fiber Optics, Elsevier Inc. 2007.

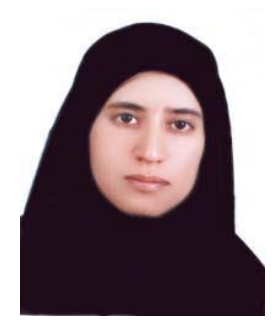

Elham Yousefi was born in Shiraz, Fars, Iran. She received her B. Sc. and M. Sc. and Ph.D in Atomic and Molecular Physics from Qom University, Alzahra University and Yazd University in 2004, 2010 and 2015, respectively. Her main research interests include: Nonlinear Optics, Multi-level Integrated Optics and Solar cells. 


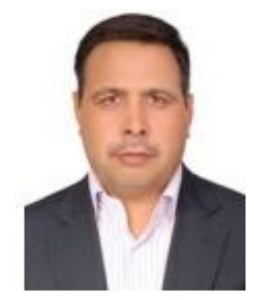

Mohsen Hatami was born in Abadeh, Fars, Iran. He received his B. Sc., M. Sc. and Ph.D degrees in Nuclear Physics, Theoretical Physics and Atomic Physics (Optics and Lasers) from Shiraz University, Shiraz, Iran in 1990, 1993 and 2006 respectively. He became an Associate Professor in 2011 at Yazd University. From 2008 to 2012 he was the head of Atomic and Molecular Physics at Yazd University. At now he is an academic staff in Shiraz University of Technology. His current research interests include: Nonlinear Optics, Optical Soliton, Integrated Optics and Photonics. Dr. Hatami is a member of the
Optics and Photonics Society of Iran (OPSI) and Physics Society of Iran.

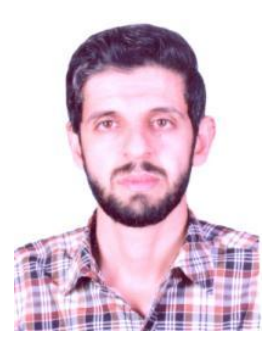

Sajjad Dehghani received his B.Sc. and M.Sc. degrees from Shiraz University and Ph.D from Tarbiat Modares University in Electronic Engineering, in 2002, 2006 and 2012 respectively. He is an assistant professor in Electronic Engineering at Shiraz University, Shiraz, Iran. His current research interests include: Nanoelectronics, Optoelectronics, Thin film Solar cells, Gas sensors, Nanocoatings. 
THIS PAGE IS INTENTIONALLY LEFT BLANK. 